A. Pramesh Rao, G. Swarup and Gopal-Krishna, eds.

\title{
Towards a Concept Design for a LOFAR
}

\author{
Jaap D. Bregman \\ Netherlands Foundation for Research in Astronomy, AA Dwingeloo, \\ The Netherlands
}

\begin{abstract}
The LOw Frequency ARray is a Digital Software Radio Telescope under study by the NFRA and the NRL. A scaleable architecture is proposed for the antenna stations of an aperture synthesis array operating in the $10-160 \mathrm{MHz}$ frequency band. The use of advanced phased array technology with digital beamforming results in a multi-beam capability of up to 64 beams, which cover together about a steradian on the sky, and can be placed such that all relevant science objects are covered simultaneously. Based on the fast expansion of high performance processing technology, it is just after 2003 that signal and data processing will no longer dominate the cost of LOFAR in producing a confusion limited sky survey at the mJy level.
\end{abstract}

\section{Basic Features of LOFAR}

Recent developments in the area of signal processing and ionospheric modelling indicate that long standing scientific challenges in radio astronomy can now be addressed (Kassim \& Erickson 1998). This triggered initiatives to study a novel wide band low frequency array with large collecting area (Bregman 1999). Bandwidth synthesis in combination with aperture synthesis of an exponentially expanding array provides complete $\mathrm{U}, \mathrm{V}$-coverage for continuum sources, which forms the basis for thermal sky noise limited performance. Such an array consists of order 40 stations, separated up to $300 \mathrm{~km}$. Although all possible beams on the sky are formed at each phased array antenna station only the signals of a limited subset of these beams are cross-correlated between all stations.

The antenna stations have a structure with four scaled fractal rings each with 64 active short dipoles. Each ring is optimized for an octave of frequency range providing a beamwidth of $1 / 8$ radian and has $50 \%$ effeciency for the next lower octave. Over the four octave frequency range an average aperture efficiency of $50 \%$ is obtained for the full station, which is comparable to that of dish type telescopes. Alternatively, all four rings can be tuned independently to their optimum frequency providing four times $25 \%$ of the full array. With a total of about 10,000 dual polarisation receptors, an effective collecting area of $1 \mathrm{~km}^{2}$ is obtained at a frequency of $10 \mathrm{MHz}$.

The fractally sparse configuration of a single ring gives side-lobes covering a hemisphere typically at $1 \%$. This has as a consequence that the self calibration procedure needs to correct for about five times as many objects in these side lobes as in the main lobe. The calibration and interference handling strategy 
has three levels, spectral filtering at the receptor level, spatial filtering and subtraction with the adaptive beam-formers at the station level, using voltage Self-cal and spatial filtering at the array level after correlation using Self-cal.

The F3X architecture extends the principles of the well known spectral Fourier transform correlator to the spatial domain. Per station we do not only make a spectral FFT but also a spatial FFT to form all possible beams on the sky. By cross-correlating a beam with a strong interfering signal with all the receptors and all the beams we can perfectly self calibrate each station and completely subtract that interference from all beams. A key element in the hardware architecture is the transposition of spectral data streams per receptor into receptor streams per frequency channel. This brings all signals that need to be combined by a beam-former together in a single serial data stream to be fed into a processor cluster that has sufficient power to process that data in a time which is the inverse of the channel bandwidth. This bandwidth is easily controlled by proper selecting the frequency resolution of the spectral FFT per receptor, which has fortunately hardly any processing power penalty. In this way not only the signal routing is solved, but scaleability is created to match to the performance of actual devices.

In a digital phased array we need to make an optimum distribution of the available processing power over the various signal and data processing tasks. Since the processing requirements of spectral filtering, beam-forming, adaptive nulling, correlation, convolution and Self-calibration, which are of comparable magnitude, all scale differently with observing frequency some flexibility is needed to match to the needs of an observation within the four octave range.

In conclusion, the proposed instrument will provide unique capabilities in terms of spatial resolution and sensitivity, not available anywhere in the world. The proposed antenna station concept with four scaled fractal rings is, depending on available budgets and interest, expandable to broader range from 10$350 \mathrm{MHz}$.

Multi-beam observing greatly simplifies telescope scheduling since all objects proposed by different astronomers can be observed simultaneously. For each frequency band this effectively extends the integration time per object from about a third of a day to order months. Multi-beaming allows to reduce the investment in receptors and signal transmission bandwidth, just by proper distribution of signal and data processing power. As a consequence we have to rely heavily on the self-calibration algorithms in AIPS++ to remove any artifacts in a single map as not to show up when hundreds of such maps are averaged to acquire the ultimate sensitivity.

\section{References}

Kassim, N. E., \& Erickson, W. C. 1998 in Proc. of SPIE, 3357, 740-754

Bregman, J. D. 1999 in Proc. of NFRA SKA Symposium: Technologies for Large Antenna Arrays, Dwingeloo

Bregman, J. D., Tan, G. H., Cazemier, W., Craeye, C. 2000 in Proc. of IEEE International Symposium on Antennas and Propagation, Salt-Lake City 